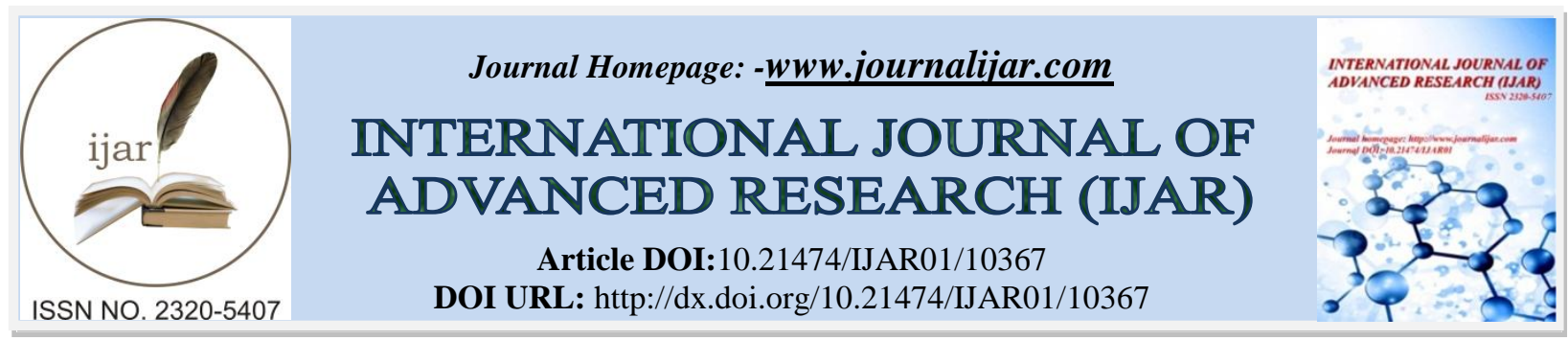

RESEARCH ARTICLE

\title{
EVALUATION OF PREANALYTICAL ERRORS USING QUALITY INDICATORS: EXPERIENCE IN A TERTIARY CARE CENTRE
}

1. Ram Manohar Lohia Hospital.

Saswati Das ${ }^{1}$ and Kajal Nandi ${ }^{2}$

2. Hamdard Institute of Medical Sciences and Research.

\section{Manuscript Info}

Manuscript History

Received: 30 November 2019

Final Accepted: 31 December 2019

Published: January 2020

\begin{abstract}
In the past few decades major revolution has been observed in the field of biochemical laboratory testing. Laboratory medicine has evolved as a science and plays a pivotal role in the provision of healthcare to the population and hence there is an ever increasing demand for reliability and accuracy of the lab tests. The quality of testing by a clinical laboratory plays a significant role in the field of health care. There are many different factors that contribute to accurate test results in the biochemistry laboratories. These factors can be classified into three phases: pre-analytical, analytical and post-analytical. Pre-analytical phase is the major source of mistakes in a clinical laboratory. Quality indicators (QIs) are therefore required to cover all steps in the preanalytical phase, from test requisition to sample storage. In this study pre-analytical errors in a clinical chemistry laboratory were analyzed over a period of 6 months.

Copy Right, IJAR, 2020,. All rights reserved.
\end{abstract}

\section{Introduction:-}

The pre-analytical phase contributes $70 \%$ of total errors in the total testing process.(1) The most commonly reported pre-analytical errors are: a) missing sample and/or test request, b) wrong or missing identification, c) contamination from infusion route, d) haemolysed, clotted, and insufficient samples, e) inappropriate containers, f) inappropriate blood to anticoagulant ratio, and g) inappropriate transport and storage conditions. (2) The 2012 ISO 15189 standard "Medical laboratories: Particular requirements for quality and competence" establishes that the preanalytical phase of the testing process begins with the test request from the healthcare provider and includes the requisition, preparation of the patient, collection of the primary sample and transportation of the sample to and within the laboratory. The preanalytical phase ends when the analytical examination begins. Clause 4.12.4 of this standard, which is used for medical laboratory accreditation, requires the implementation of quality indicators for systematically monitoring and evaluating the contribution of the laboratory to patient care and the identification of improvement opportunities.(3) Quality indicators (QIs) are fundamental tools enabling users to quantify the quality of laboratory services. (4) In the recent years there has been increasing interest among healthcare professionals in quality assurance in preanalytical phases of laboratory testing. The International Federation of Clinical Chemistry and Laboratory Medicine (IFCC) Working Group on Laboratory Errors and Patient Safety (WG-LEPS) has made an important contribution to developing QIs for the preanalytical phase and specifications for those indicators.(5-7)

Corresponding Author:-Saswati Das

Address:- Ram Manohar Lohia Hospital. 
Every laboratory should design a policy for detection and prevention of errors. The frequency of errors should be determined systematically in a standardized manner. In this study we assess the preanalytical process and analyze each error in the preanalytical phase, and how the most common errors can be determined.

\section{Methodology:-}

This was an observational study conducted at Lok Nayak Jai Prakash Hospital which is a tertiary care hospital in New Delhi . This study was conducted for a period of 6 months during January 2016 - June 2016. The hospital has both broad specialty and superspeciality departments. The central laboratory of the hospital is comprised of three departments biochemistry, pathology and microbiology. The clinical chemistry laboratory receives monthly approximately 6000 test requisition forms and 9500 blood samples from inpatient and outpatient departments. The clinical chemistry lab is equipped with autoanalyzers DXC 800 (Beckman Coulter, USA), Olympus AU 400 \& Olympus AU 480 (Beckman Coulter, USA) for routine blood investigations. The biochemistry lab also has three automated Direct ISE Ecolyte analyzer by Cobas for electrolyte measurement and a Biorad D10 for glycated haemoglobin estimation. The clinical chemistry department has separate test requisition form which includes patient details, disease diagnosis, sample information and investigations. All blood samples were collected in vacutainers and transported in icepack to the central lab. On receiving the sample in lab, technical staff checked for any preanalytical errors in the test requisition form(TRF) and in the sample. If the sample did not meet the standards and the TRFs had missing information, the sample was rejected and the concerned hospital staff was informed. An entry was made in the log book for every rejected sample citing the reason for rejection. The preanalytical errors included in the study are, patient details like name, age, sex, date, registration number (OPD/IPD number), provisional diagnosis/ short clinical history, sample routine/emergency (stat), errors in test input and signature of treating physician in the test requisition forms. Blood samples were observed for hemolysis, lipemia, improper blood to anticoagulant ratio, collection in wrong vacutainer, missing samples, mislabelled samples, improper transportation, insufficient sample for processing, improper storage and phlebotomy errors. Any error during sample collection was also recorded in inpatient wards and OPD collection centre. In this study nineteen prenalytical quality indicators designed based on (IFCC) Working Group on Laboratory Errors and Patient Safety (WG-LEPS) recommendation (7), were assessed monthly for 6 months to monitor the preanalytical phase Nine of them pertaining to errors in the test requisition forms and ten QIs to track sample collection and transport. All the data was collected from the logbooks of the inpatient wards, laboratory and OPD collection centre and entered in an excel sheet. Microsoft Excel was used for the analysis of the data.

\section{Results:-}

The present study was conducted over a period of 6 months. A total of 64,652 samples and 39,471 test requisition forms (TRF) were screened for preanalytical errors from clinical biochemistry section. Among the 64652 Test requisition forms 26375 were from out patient department(OPD) and 38277 from the inpatient wards . A total of 1645 samples from OPD and 865 samples from inpatient wards of different departments having preanalytical errors and were rejected during the 6 month period.

The frequency of preanalytical quality indicators to evaluate test requisition forms is shown in Table 1 and the frequency of preanalytical quality indicators to evaluate sample collection and transport is depicted in Table 2.

Table 1:- Preanalytical Quality Indicators to analyze test requisition forms(n=39,471).

\begin{tabular}{|l|l|l|l|}
\hline Serial No. & Preanalytical Quality Indicator & Number of errors & Frequency (\%) \\
\hline 1 & Age & 2379 & 6.03 \\
\hline 2 & Gender & 1921 & 4.87 \\
\hline 3 & Date of collection & 1535 & 3.89 \\
\hline 4 & Registration number. (OPD/IPD) & 3733 & 9.46 \\
\hline 5 & Diagnosis not mentioned & 25,676 & 65.05 \\
\hline 6 & Routine/Emergency & 5274 & 13.36 \\
\hline 7 & Type of specimen & 6141 & 15.56 \\
\hline 8 & Treating Physician Details and Signature & 16,785 & 42.52 \\
\hline 9 & Errors concerning test Input/entry & 1374 & 3.48 \\
\hline
\end{tabular}


Table 2:- Preanalytical Quality Indicators to analyze sample collection \& transportation ( $\mathrm{n}=64,652)$.

\begin{tabular}{|l|l|l|l|}
\hline Serial No. & Preanalytical Quality Indiacator & Number of errors & Frequency (\%) \\
\hline 1. & Insufficient sample volume & 259 & 0.40 \\
\hline 2 & Inappropriate Sample transportation & 127 & 0.20 \\
\hline 3 & Inappropriate Sample Storage & 74 & 0.11 \\
\hline 4 & Hemolyzed Sample & 472 & 0.73 \\
\hline 5 & Lipemic Sample & 165 & 0.26 \\
\hline 6 & Collection in a wrong vacutainer & 668 & 1.03 \\
\hline 7 & Inadequate sample to anticoagulant ratio & 276 & 0.43 \\
\hline 8 & Mislabelled samples & 382 & 0.59 \\
\hline 9 & Samples lost/not received & 411 & 0.64 \\
\hline 10 & Phlebotomy errors & 87 & 0.13 \\
\hline
\end{tabular}

\section{Discussion:-}

Laboratory results are paradigmatic for the diagnosis and management of diseases.(8-10) Major focus of the laboratories is maintaining the accuracy of the analytical phase of the testing process. The preanalytical phase, as well as the post-analytical phase hold equal importance. The preanalytical phase is riddled with many issues like improper filling of the requisition forms, lack of staff training about ideal phlebotomy procedures etc. Several Studies (11-14) have shown that most incorrect results originate in the preanalytical phase. Preanalytical errors account for more than $70 \%$ of the total number of laboratory errors and have a significant clinical and economic impacts on medical care. Therefore the preanalytical phase must be under strict supervision at all times so that the laboratory quality reaches a benchmark. Quality indicators are essential performance indicators for the preanalytical phase of the testing process.

The major pre-analytical errors of concern noticed in our study pertaining to the test requisition forms was that the treating physician's details were missing in $42.52 \%$ of the forms and the provisional diagnosis/history were missing in $65.05 \%$ of the lab forms. Diagnostics today is not merely sample processing and dispatch of reports. The "brain to brain loop" needs closing. One of the good laboratory practice is disseminating information about critical results to clinicians so that corrective measures can be initiated for the patient at the earliest. Incomplete patient information is a major challenge to patient safety and leads to loss of precious time. The critical results in $37 \%$ patients could not be communicated to the treating physicians due to lack of the details of the treating physician. In some of the TRFs, missing patients information was tracked by our staff by connecting with the staff and doctors of inpatient wards, however this impacted the turn around time. In case of OPD patients the TRFs citing the reason for sample rejection, were dispatched to the OPD for the complete information. The tests mentioned for the rejected specimens had to be repeated with fresh samples and new test requisition forms when the patients visited the hospital next time. The followup in such cases was very difficult. These errors can be minimized by persistence of laboratory for complete information on TRFs and sincere efforts by the physicians \& hospital staff to provide the same.

Each analytical process requires a fixed volume of serum/plasma for analysis. A major finding in our study was errors during sample collection (insufficient sample volume- $0.4 \%$, collection in wrong vaccutainer $-1.03 \%$, hemolyzed samples-0.73\%). In case of a hemolyzed sample often a fresh sample for processing the tests was requested leading to an increase the turn around time. In our study lipemia accounted for rejection of $0.26 \%$ of the samples in the inpatient and outpatient departments respectively. This can be avoided by proper patient preparation, preferably after an overnight fast. If the patient has any kind of metabolic disorder or is a known case of dislipidemia, the same must be mentioned in the requisition slip. Many patients who do not follow the patient preparation instructions properly and give samples in non-fasting states leading to erroneous reporting. It is the responsibility of the physician and the phlebotomists to ensure that proper patient preparation instructions is disseminated and adhered to before sample collection. Our data is comparable to data provided by other investigators in India which confirms that problems directly related to specimen collection are the main cause of preanalytic errors, especially hemolyzed, clotted, insufficient, and incorrect samples (15-18). Incorrect phlebotomy practices is one of the major reason behind preanalytical errors in sample collection. The main reasons being lack of training of the phlebotomists and difficulty of sampling as in patients whose veins are difficult to localize(e.g. paediatric, chemotherapy patients). The institute has a centralized collection center where samples for clinical biochemistry, hematology, microbiology, are collected simultaneously. Regular in-house training sessions are 
organized by experts for the technicians to familiarize them with the standard operating procedures(SOPs) for sample collection \& processing. These training programs facilitated the adoption of ideal phlebotomy practices by our laboratory personnel. The samples are transported to our laboratory from the collection center by the staff following the SOP that must be adhered to during transport. Monitoring the QIs in the preanalytical phase had a major impact in our laboratory service as each step was under constant surveillance leaving little room for error.

The limitation of our study is that some pre-analytical variables like patient preparation, diet and drug intake history of the patient, timing of sample collection have not been included as this is a hospital based laboratory where samples are received from patients attending OPD or admitted in IPD. In our study only those pre-analytical variables were included which were under direct supervision of lab personnel.

\section{Conclusions:-}

Total quality management includes all the steps involved in sample processing, beginning from test ordering to the final interpretation of results by the clinicians. Detecting errors at all stages of analysis, evaluating them and devising corrective measures for their prevention can make a major impact on the quality standard of the laboratory. Identification of QIs in all phases of testing an essential step in assuring quality in total testing process and patient safety. QIs are formidable tool for identifying the risk of errors during the critical steps of the preanalytical phase. Encouraging ideal phlebotomy practices and sample transport procedures is quintessential for efficient laboratory functioning. As laboratorians, we need to adopt a comprehensive approach towards laboratory diagnosis and communicate well with the clinicians in order to provide better patient services.

\section{References:-}

1. Lippi G, Chance JJ, Church S, Dazzi P, Fontana R, Giavarina D, et al. Preanalytical quality improvement: from dream to reality. Clin Chem Lab Med 2011;49:1113-26.

2. Carraro P, Plebani M. Errors in a stat laboratory: types and frequency 10 years later. Clin Chem 2007;53:133842.

3. International Organization for Standardization. ISO 15189:2012:medical laboratories:particular requirements for quality and competence. Geneva, Switzerland: InternationalOrganization for Standardization; 2012.

4. Plebani M. Quality indicators to detect pre-analytical errors in laboratory testing.ClinBiochem Rev. 2012;33:85-8

5. Sciacovelli L, Plebani M. The IFCC working group on laboratory errors and patient safety.ClinChimActa. 2009;404:79-85.

6. Plebani M, Sciacovelli L, Lippi G. Quality indicators for laboratory diagnostics: consensus is needed. Ann ClinBiochem. 2011;48:479.

7. Sciacovelli L, O'Kane M, Skaik YA, et al. Quality indicators in laboratory medicine: from theory to practice. Preliminary data from the IFCC Working Group Project "Laboratory Errors and Patient Safety".ClinChem Lab Med. 2011;49:835-44.

8. Westgard JO, Darcy T. The truth about quality: medical usefulness and analytical reliability of laboratory tests. ClinChimActa. 2004;346:3-11.

9. Plebani M. The future of clinical laboratories: more testing or knowledge services? ClinChemLab Med. 2005;43:893-96.

10. Regan M, Forsman R. The impact of the laboratory on disease management. Dis Manag. 2006;9:122-30.

11. Plebani M, Ceriotti F, Messeri G, Ottomano C, Pansini N, Bonini P. Laboratory network of excellence: enhancing patient safety and service effectiveness. Lab Med.2006;44:150-60.

12. Bonini P, Plebani M, Ceriotti F, Rubboli F. Errors in laboratory medicine. Clin Chem. 2002;48:691-98.

13. Wiwanitkit V. Types and frequency of preanalytical mistakes in the first Thai ISO 9002:1994 certified clinical laboratory, a 6-month monitoring. BMC Clin Pathol. 2001;1:5.

14. Nutt L, Annalise EZ, Rajiv TE. Incomplete lab request forms: the extent and impact on critical results at a tertiary hospital in South Africa. Ann Clin Biochem. 2008;45:463-6.

15. Chawla R, Goswami B, Singh B, Chawla A, Gupta VK, Mallika V. Evaluating laboratory performance with quality indicators. Lab Med. 2010;41:297-300.

16. Chillar N, Khurana S, Agarval R, Singh NK. Effect of pre-analytical errors on quality of laboratory medicine at a neuropsychiatry institute in North India. Indian J Clin Biochem.2011;26:46-9.

17. Gajjar M, Patel A, Jain S. Monitoring of quality indicators in pre analytical phase of testing in the clinical biochemistry laboratory of a tertiary care hospital attached with government medical college. IOSR-JDMS. 2016;15(7):62-8. 
18. Kulkarni S, Ramesh R, Evaluation of Preanalytical Quality Indicators by Six Sigma and Pareto's Principle. Ind J Clin Biochem (Jan-Mar 2018) 33(1):102-7. 